University of South Florida

DIGITAL COMMONS

@ UNIVERSITY OF SOUTH FLORIDA
Digital Commons @ University of

South Florida

3-1-2005

\title{
Fixed Route Transit Scheduling in Florida: The State of the Industry
}

CUTR

Follow this and additional works at: https://digitalcommons.usf.edu/cutr_nctr

\section{Scholar Commons Citation}

CUTR, "Fixed Route Transit Scheduling in Florida: The State of the Industry" (2005). Research Reports. 231.

https://digitalcommons.usf.edu/cutr_nctr/231

This Technical Report is brought to you for free and open access by the National Center for Transit Research (NCTR) Archive (2000-2020) at Digital Commons @ University of South Florida. It has been accepted for inclusion in Research Reports by an authorized administrator of Digital Commons @ University of South Florida. For more information, please contact digitalcommons@usf.edu. 


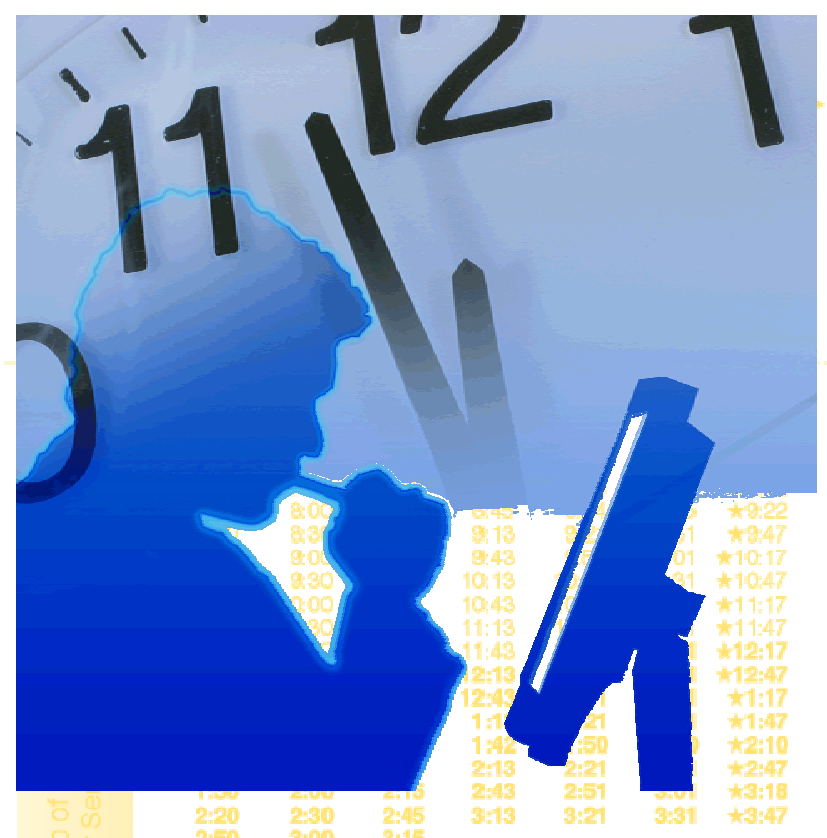

Fixed Route Transit Scheduling in Florida:

The State of the Industry

Final Report 


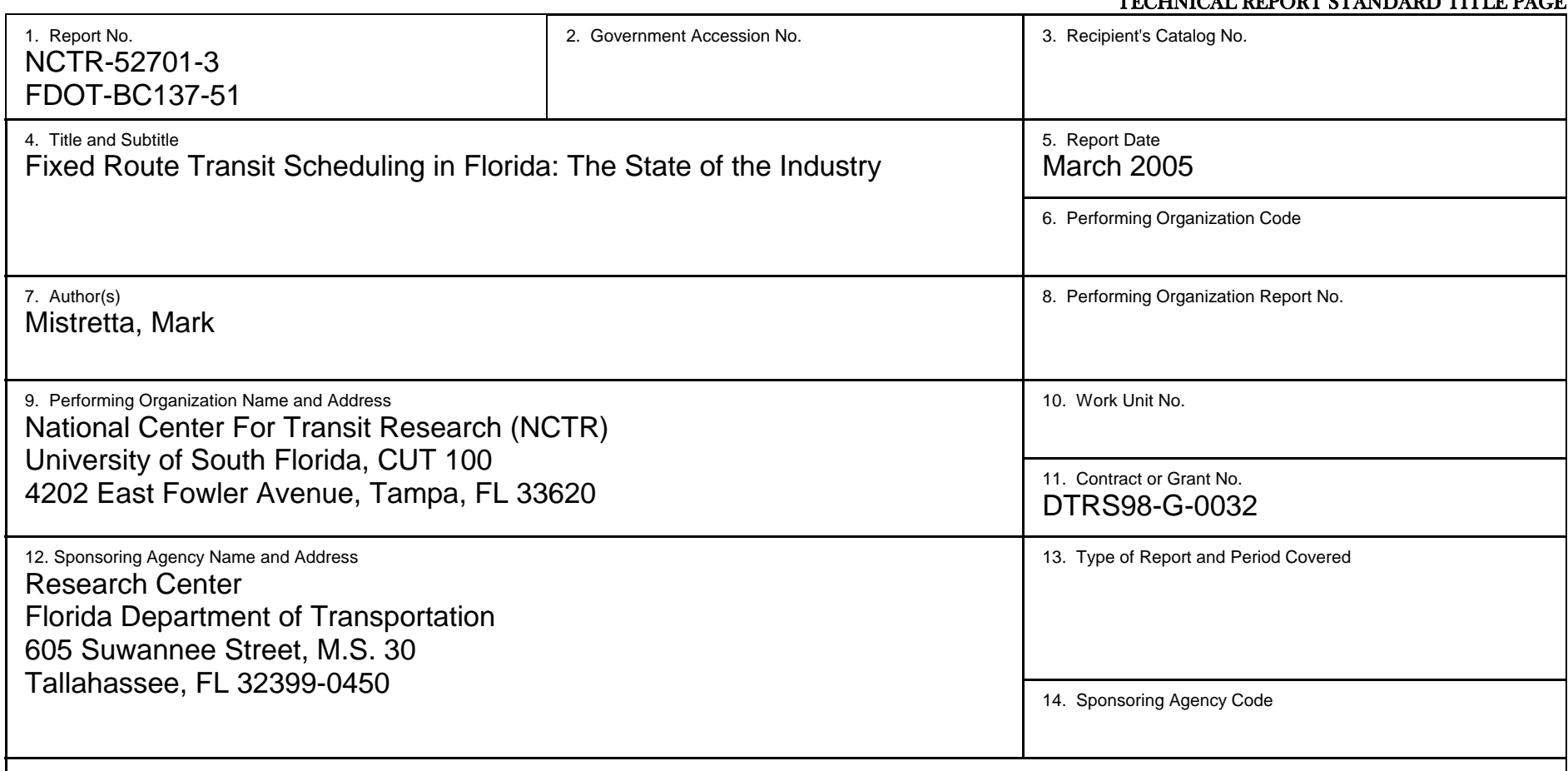

15. Supplementary Notes

A project in the Public Transportation Synthesis Series

16. Abstract

Fixed route transit scheduling is an undervalued and often misunderstood task of public transit agencies. It is a complex process that involves several detailed analytical procedures. Trip building, blocking, runcutting, and rostering are all sub-tasks of the overall task of scheduling. This project examines the basic framework of the scheduling process to provide a general understanding of the subject.

The main objective of this project was to examine current scheduling practices at transit systems in Florida and assess each agency's scheduling issues and potential need for technical assistance in schedule development. A scheduling survey was designed and distributed to all Florida fixed route transit systems. The results of this survey are presented and analyzed with a specific emphasis on comparing systems that use automated scheduling software versus those that continue to schedule using manual processes.

The use of automated scheduling software at the larger transit systems in Florida has yielded various improvements to their scheduling process. Scheduling software is an iterative tool that provides the scheduler with greater flexibility, functionality, and control over scheduling their services. It also works to reduces mistakes, improve vehicle and operator efficiencies, reduce staff time on tedious activities, and provide better reporting capabilities.

As reflected in the fixed route scheduling survey, there is an interest among small to medium sized transit systems in realizing the benefits of automated scheduling software. However, scheduling software costs and computer hardware costs in general were seen as the most prohibitive aspects of attaining scheduling software. Furthermore, the learning curve and extensive set-up time required is a potential obstacle for small to mid-sized systems.

Based on the findings of this report, a mechanism could be explored that would enable small to medium sized transit systems in Florida the opportunity to provide more efficient services through automated scheduling.

17. Key Words

Transit, Scheduling, Software
18. Distribution Statement

Available to the public through the National Technical Information Service (NTIS), 5285 Port Royal Road, Springfield, VA 22161, (703) 605-6000, http://www.ntis.gov/ and through the NCTR web site at http://www.nctr.usf.edu/

20. Security Classif. (of this page) Unclassified

\begin{tabular}{l|l}
$\begin{array}{l}\text { 21. No. of pages } \\
36\end{array}$ & 22. Price \\
\hline
\end{tabular}




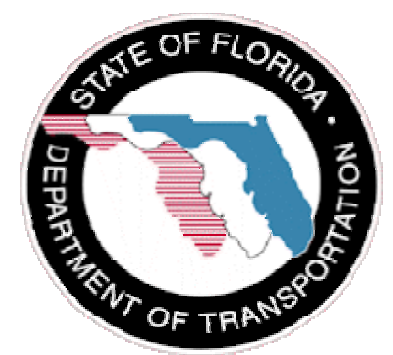

\title{
State of Florida Department of Transportation
}

Public Transit Office

605 Suwannee Street

Tallahassee, FL 32399-0450

(850) 414-4500

Project Manager: Tara Bartee, Planning Administrator
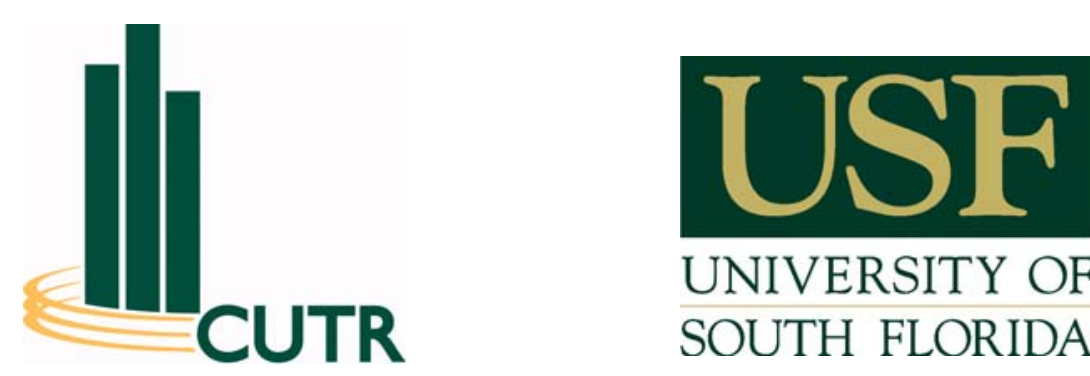

UNIVERSITY OF SOUTH FLORIDA

\author{
National Center for Transit Research \\ Center for Urban Transportation Research \\ University of South Florida \\ 4202 E. Fowler Avenue, CUT 100 \\ Tampa, FL 33620-5375 \\ (813) 974-3120
}

Project Directors: Joel Volinski, NCTR Director

Dennis Hinebaugh, Transit Program Director

Project Manager: Mark Mistretta, Research Associate

Project Staff: Chris Deannutis, Research Associate

The opinions, findings and conclusions expressed in this publication are those of the authors and not necessarily those of the U.S. Department of Transportation or the State of Florida Department of Transportation. 


\section{Table of Contents}

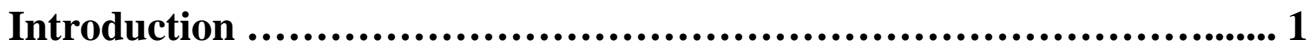

The Scheduling Process ....................................................... 3

Trip Building

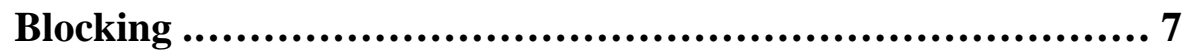

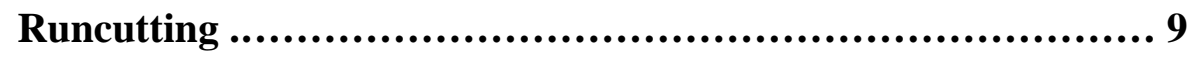

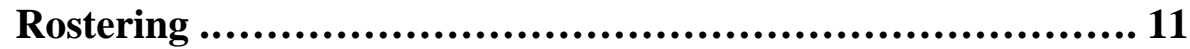

Scheduling Survey of Florida Transit Agencies

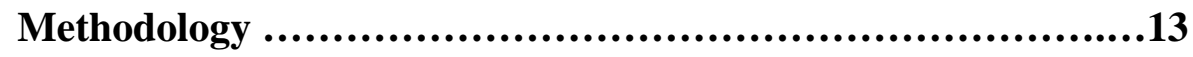

Results ........................................................ 13

Application Overviews:

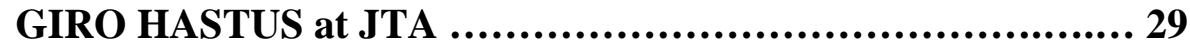

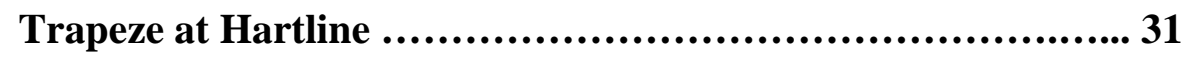

Fleet-Net at RTS .............................................. 34

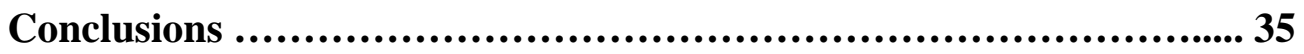

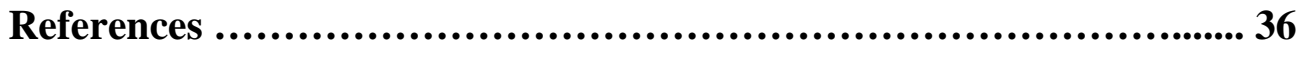

Appendix A (Fixed Route Scheduling Survey) ....................... A1 


\section{Introduction}

Fixed route transit scheduling is an undervalued and often misunderstood task of public transit agencies. It is a complex process that involves several detailed analytical procedures. Trip building, blocking, runcutting, and rostering are all sub-tasks of the overall task of scheduling. Throughout the process, there are various inputs that shape and define the procedures requiring a scheduler to constantly reassess and alter the outputs. Symbolically, transit scheduling can be compared to designing a jigsaw puzzle: painting the picture (trip building), cutting the puzzle into pieces and taking it apart (blocking and runcutting), and putting it back together again (runcutting and rostering).

The manual scheduling process has become a highly specialized field over the history of fixed route bus transit in North America and more specifically the State of Florida. Typically, one or two persons per transit system were given the assignment of scheduling for their systems. These transit schedulers have been entrusted with providing highly efficient and workable solutions that satisfy local labor agreements and agency rules.

The arrival of the computer age in the 1980's has ushered in a host of new software programs to assist the transit scheduler in his tasks. This automated scheduling software provides another more advanced method for scheduling fixed route transit services. This software enables agencies to set up the parameters of their work rules into the program and automates certain aspects of the scheduling process that ideally provide more efficient schedules. Other potential benefits of automated scheduling include: the reduction of staff time needed for scheduling processes, cost savings of reducing vehicles or operators needed, and the flexibility and functionality inherent with computer software programs.

The main objective of this project was to examine current scheduling practices at transit systems in Florida and assess each agency's scheduling issues and potential need for technical assistance in schedule development. A scheduling survey was designed and distributed to all Florida fixed route transit systems. The results of this survey are presented and analyzed with a specific emphasis on comparing systems that use automated scheduling software versus those that continue to schedule using manual processes. A basic framework of the scheduling process is also described to provide a general understanding of the subject area. Three application overviews are presented: a review of 
Jacksonville Transportation Authority's (JTA) use of GIRO HASTUS, Hartline's (Tampa) use of Trapeze software, and Regional Transit System's (RTS) (Gainesville) use of Fleet-Net (the only agency in Florida to utilize a software package other than Trapeze or HASTUS). These three fixed route software packages encompass all of the automated scheduling software currently in use at Florida transit properties. Finally, the future potential of a state-wide scheduling resource center to assist Florida transit agencies with their scheduling needs is explored. 


\section{The Scheduling Process}

Scheduling for fixed route transit service is a highly technical activity that requires an extensive knowledge of transit terminology. Throughout this section, TCRP Report 30, "Transit Scheduling: Basic and Advanced Manuals" is referenced heavily for definitions and explanations of various transit scheduling terms. The following diagram (Figure 1) assembles these terms and displays the data flow of the various inputs and outputs of the four sub-tasks (trip building, blocking, runcutting, and rostering) in the fixed route transit scheduling process.

Figure 1 - Fixed Route Transit Scheduling Data Flow

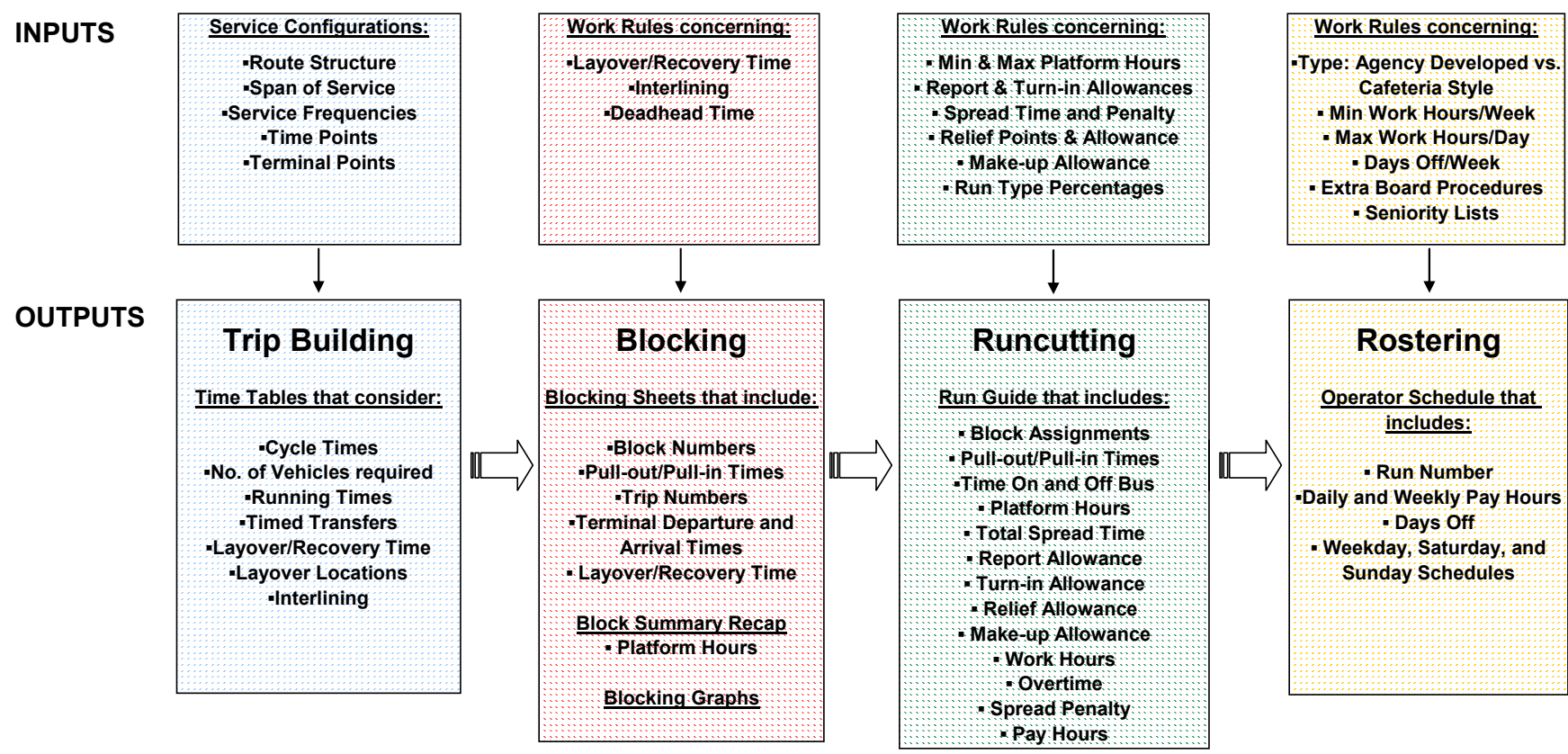

Before a transit scheduler can begin the scheduling process, basic configurations of their transit services are required. These include route structures, span of service, service frequencies, time points, and terminal points. The structure of each route in the network defines where each vehicle travels during service. The span of service defines the hours of operation for each route and is often a function of ridership demand. Service frequencies or headways define the time intervals between vehicles arriving at a particular stop. Frequencies may be policy-based (fixed intervals), or demandbased (related to passenger loads). The headway on a policy-based schedule will often be an even 
"clock multiple" such as every 10,15, 20, 30, 45, or 60 minutes. Policy based schedules are a convenience to the passengers, informing them that a vehicle will arrive at their stop every $\mathrm{x}$ minutes past the hour. With demand-based frequencies, the agency policy dictates that the level of service provided is directly related to the passenger load and the vehicles capacity to carry them. Service intervals will no longer be regular, so demand-based frequencies are typically used only when passenger loads at specific locations are so great that fixed intervals are no longer an option. Time points are the stops along a location that are used in building the route time tables. They are typically spaced 6 to 10 minutes apart and are generally located at major street intersections that possess sidewalks and traffic signals. It is also useful to locate time points at major trip generators such as shopping centers, hospitals, and government buildings, or locations where time is crucial such as employment centers and multi-modal centers. Terminal points are considered the end points of routes and are preferably located in locations that ensure operator safety and comfort. 


\section{Trip Building}

Trip building (or creating time tables) is the foundation of the scheduling process. It involves creating the "master" service schedule, which indicates all of the times that revenue service vehicles are scheduled to be at specific locations along the route to address customer service demands. The trip building process is usually managed by a specific scheduler or Scheduling Department, or a combination of Planning and Operations Departments. Other departments, such as Marketing and Finance may contribute information to the process.

Various characteristics that are considered outputs of the trip building process include cycle times, layover and recovery time, number of vehicles required per route, timed transfers, running times, and interlining considerations. Cycle time measures the total time it takes a bus to make a round trip on the route and is a required measure for building a master schedule. Cycle time also includes an amount of time for layover and recovery time. Layover time can be defined as rest or "break" time allocated to the operator. The minimum amount of layover time is usually dictated by labor agreement, agency standards, or past practice. Recovery time is "buffer" time built into the schedule that may or may not be needed by the operator. If the vehicle is behind schedule, the recovery time allows it to catch up to its schedule at the end of the line. While recovery time and layover time are often taken at the same location, recovery time is distinct and likely allocated at the discretion of the agency. The total cycle time is used to determine the number of vehicles required to operate each route on the desired headway. For example, if the cycle time of a route is 90 minutes and the desired headway is 30 minutes, then the route will require three vehicles in operation. Maintaining a fixed interval frequency will sometimes lead to excessive recovery time assigned to a route. When this occurs, a scheduler can recommend several options to the Service Planning Department or Management. The headway could be made shorter, the route could be lengthened to serve a larger area, the route could be deviated, or the route could be interlined with another route.

Timed transfers require that vehicles converge at a common location at approximately the same time, so that passengers can depart one vehicle and board another. Schedules are often designed such that timed transfers take place at the most heavily used locations to minimize the wait time for the most passengers. If an agency uses timed transfers, those locations will dictate the remainder of the affected routes schedules. Timed transfers may also result in the need for extra layover or recovery time so that trips arrive and leave at the same time. Therefore the scheduler has to constantly balance 
the master schedule between the often opposing goals of cost effectiveness and responding to the customer needs.

Whether using manual or automated scheduling, the process of creating the master schedule for a transit system requires collection of spatial and temporal data. A majority of systems use Operation Supervisors or Planners who go out "in the field" to determine time points, define route structure, and measure running times. Running time refers to the number of minutes assigned to a revenue vehicle for moving from one time point to the next. When measuring running times, an agency may utilize a car or van, but it can be more accurate if an actual bus is used to better simulate the actual operating environment. Systems who utilize Automatic Vehicle Location (AVL) systems possess an even better, more accurate way of calculating running times as they can base their schedules on actual travel times between time points. Regardless of the method, accurate data collection is an essential factor in building an effective transit schedule. If too little time is provided in the route schedules, then operators will continuously run late and provide unreliable service. Another potential effect of too little time is rushed or frustrated operators who may endanger passenger safety or neglect customer service by attempting to remain on an impossible schedule. If too much time is provided, then the service is not making efficient use of the agency's equipment and operators and creates longer travel times for passengers. Furthermore in this scenario, operators may run "hot" or ahead of schedule which is considered worse than running late as it can cause customers to miss their trips altogether. A best practice is to measure running times as accurately as possible, match the schedule accordingly, and provide adequate layover and recovery time at the end of the line.

Interlining is another consideration during the trip building phase of the scheduling process. It involves scheduling a vehicle to switch from one route to another route during a service day. It is a practice that a transit system can utilize that may result in reduced costs to the agency and provide a convenience to the passenger. Additional potential benefits include the elimination of end-of-line looping, reduction of excessive layover or recovery time, and the reduction of passenger transfers from vehicle to vehicle. Transit agencies often have policies that define the amount and location of interlining that can be scheduled.

The final result of the trip building task will be a set of time tables for each route that provides the time at which every vehicle in the system is at the defined time points. For an agency converting from manual scheduling to automated scheduling software, the initial set-up of relevant data in the 
program is the most difficult and time-consuming task and occurs both before and during the trip building task. The majority of this data can be pulled from existing data sets, but the set-up process will still require a solid understanding of how the software works and a considerable amount of time for data entry. Agencies that make the conversion to automated software often have a long adjustment period for training and learning the intricacies of the programs.

\section{Blocking}

After a "master" service schedule is established, the next step in the scheduling process is blocking. Blocking involves creating assignments that describe the activity for a single vehicle for a single service workday. These assignments often contain several vehicle trips that are linked together as part of the block. Each block may cover more than one route and usually involves more than one operator during the course of one day.

Blocking is a critical element in the scheduling process because it serves as the basis for revenue and non-revenue vehicle operating costs as well as influencing labor costs. As in the trip generation process, there may be agency policies that affect the blocking process. Three policies that greatly affect the process are layover/recovery time, layover locations, and interlining.

For optimal blocking, it is important to know whether the layover/recovery policy represents a guideline, or is a hard and fast rule which must be followed even if it impacts blocking efficiency. One commonly used policy requires a minimum layover and recovery time of 10 percent of the total cycle time.

Layover time can become a factor, because it must be taken in a safe location where a vehicle can stand still without impeding traffic and provide a safe resting location for operators. Layover and recovery time is generally taken at the end points of the route where it will inconvenience the fewest passengers. However, if the end points are considered to be inadequate layover locations, then another point on the route must be used, which may impact the overall scheduling process.

The vehicle blocking process can be an extremely complex task. The scheduler must have a firm grasp of the complexities of their work rules and their bus network. In addition to the agency policies listed above, there is a multitude of other possible factors that complicate the blocking process. 
Multiple terminals, variable running times, headway variations between peak and non-peak service, and "extra" trips for school service are just a few examples of factors that may have an influence.

There are two main goals of the blocking process: to optimize the number of vehicles required to provide the scheduled service and to minimize the amount of deadhead time. Deadhead is the time it takes a vehicle to travel from the garage to the start of revenue service (in customer use). Deadhead also refers to the time it takes the vehicle to return to the garage after serving its final stop on its last run. The amount of deadhead time can be reduced by limiting the number of times that vehicles must travel back to the garage and by limiting the distances the vehicle has to travel from the garage to its first time point and from its last time point back to the garage.

The actual manual blocking process requires the use of blocking sheets (likely in a spreadsheet program). The blocking sheet is a tool to help the scheduler document the trip start and end times associated with each vehicle. Information that is entered into the blocking sheet includes: the block number, the pull-out and pull-in times, the trip numbers, the departure and arrival times at the terminal points, and layover plus recovery time which are used to determine the time the trip can be hooked to another trip. A common blocking number convention is to assign a four digit number to each block with the first two digits defining the route number and the last two digits for the block number. Pull-out refers to the time that an operator must leave the garage in order to be at the first time point of the route on schedule. Pull-in is the time the vehicle is due back at the garage after revenue service ends. It is helpful to denote the trip numbers on the blocking sheet and the master schedule to ensure that all trips are blocked.

A block summary recap for each route can be used to summarize all blocks created for that route by showing only the first and last trip of each block. From this recap, the total platform hours can be calculated for each route. Platform hours measure the total time during which an operator is behind the wheel of a vehicle in both revenue and non-revenue service and is a key statistic used during runcutting.

Graphing the blocks is another valuable tool that later assists in the runcutting process. A feature of scheduling software is its capability to graph blocks automatically. Automated scheduling software also has the capability of simultaneously considering all blocking possibilities and selecting the most 
optimal solution. This process takes the software only a fraction of the amount of time it requires for a scheduler to block the trips manually using blocking sheets, much less find an optimal solution.

\section{Runcutting}

The third process in scheduling transit service is runcutting. It can be defined as the process of assigning drivers to vehicles. These assignments, also known as "runs", are assembled or "cut" from the vehicle assignments (blocks). Blocks can be cut and assembled in such as way as to create either straight runs or split (multi-piece) runs. Straight runs imply continuous work of a longer duration. A straight run generally consists of a single block piece of work close to eight hours or more, which is a suitable span for assigning an individual driver for a full work day. A straight run may also consist of two block pieces separated by a paid break (usually under an hour) in between. A split run generally consists of two (sometimes three) work assignments with unpaid breaks in between. The operator is considered off-duty during this time.

Runcutting is a critical task in the scheduling process because it defines the number of operators ultimately needed to operate the service reflected in the master schedule. Once again, this process must take into account any established work rules or agency policies. Typical rules include: minimum and maximum platform time, report and turn-in allowances, spread time and spread penalty, relief locations and allowance, make-up allowance, and run type percentages.

Minimum and maximum platform time refers to the allowable length of time that an operator may be scheduled to be behind the wheel of a vehicle in both revenue and non-revenue service. Platform time also includes time for operators to pull-out and pull-in. At many agencies, block pieces that cannot be assembled into runs of minimum platform length (based on work rules) are generally assigned to part-time operators or the Extra Board (a contingency of operators on stand-by who fill in for other operators).

Report and turn-in allowances refer to the length of time for operators to get their vehicle ready before pull-out and to attend to any duties after pull-in. A typical work rule may specify a ten minute report allowance and a five minute turn-in allowance. For split runs, the turn-in allowance will be associated with the last piece of work. 
Spread time is defined as the total elapsed time between the first report time and the final turn-in time of a run. A spread maximum typically applies to split runs and, depending on the union contract or state regulation, can vary between 10 and 15 hours in duration. The spread penalty for an agency violating this rule is typically a payment ranging from $1 / 2$ time to full-time pay for the amount of excess minutes.

Relief points are locations set up by the agency where relief operators report to replace operators whose shifts end at another location other than a garage. Relief points are necessary when the time that the vehicle operates in a given day is longer than one operator is capable of working. They are also required when an agency schedules split shifts. A relief allowance is typically paid to operators who are required to travel from the relief location back to the garage and vice versa.

A make-up allowance is payment for time not actually worked by an operator so that the pay time is equal to the minimum daily or weekly guarantee. For example, a run that totals seven hours and 50 minutes during a service day will be paid ten minutes of make-up time if the minimum daily work hour guarantee is set at eight hours.

Run type percentages refer to the percentage of runs that must be straight versus split as determined in the agency work rules. A typical run type percentage stipulation requires that $1 / 2$ of all runs must be straight runs; while one third of the remaining split runs must not exceed a 12 hour spread. Split shifts are generally not well liked by operators and may result in higher operating costs for agencies and therefore it is beneficial to limit their use. Automated Scheduling software can provide a legal solution that will often exceed the required run type percentage and in many cases can improve the cost efficiency of service.

All of the above mentioned work rules will have an influence on the runcutting process. With these rules in mind, a scheduler can now assemble a Run Guide. A Run Guide records the work and pay components of the various runs and guides the scheduler in reviewing the runs individually and collectively. After all runs are recorded on the Run Guide, the scheduler can now review and adjust the runs to provide a more optimized runcut. During optimization, the scheduler strives to: achieve the fewest number of runs necessary to provide the desired level of service, equalize platform time and pay hours among runs, ensure the runs conform to labor agreements and agency policies, and facilitate the calculation of accurate pay hours. The larger the number of block pieces that exist, the 
greater the number of possibilities for creating and optimizing runs. As the number of possibilities increase, the more complex and time-consuming the runcut becomes.

Similar to blocking, automated scheduling software can improve the runcutting process. After a scheduler inputs the parameters and work rules of their agency, the software is capable of considering all possible combinations and creating the most optimal runcutting solution. The program can also show if a run is not legal and exactly which rule it is violating. The scheduler then has the ability to undo the offending run or change the run definition rules and the program will instantly reclassify and recost the existing runs. This feature enables an agency to run policy scenarios to measure the impacts of proposed changes to work rules without completely recutting the runs.

\section{Rostering}

Rostering is the process of grouping daily operator runs into weekly run packages that operators are given the opportunity to "pick" or "bid" for a specified bid period (also known as the mark-up or lineup). The order in which operators bid on the runs they want is usually based on seniority. Weekly run packages or rosters sometimes consist of five 8-hour daily runs. Other agencies prefer to utilize four 10-hour daily runs instead. When part-timers are used, the weekly rosters may consist of two, three or four daily runs. For agencies having difficulty hiring and retaining operators, the use of five 10-hour daily runs is a common practice; however this will likely result in higher overtime costs. The rosters remain in effect until the next mark-up (pick, bid) which generally occur 3 or 4 times per year.

There are two basic types of rostering: operator developed (also known as cafeteria style) and agency developed. Under the cafeteria style of rostering, an operator can choose both specific daily runs and days off from a master list. An operator list is created by the agency that generally lists operator's names in seniority order and a time slot to pick their runs. Schedule and/or Operations Department staff will usually monitor the mark-up to ensure that operators pick work according to seniority and that all rules governing the process are adhered to. Certain restrictions may be enforced such as consecutive days off, mixture of run types, and a minimum number of off-duty hours required between each daily run. Cafeteria rostering is generally addressed very specifically in a labor agreement. 
Under the agency developed rostering approach, the daily runs are combined into weekly run packages by the agency before operators bid. Therefore, operators bid on weekly packages of runs and not on each individual run from a cafeteria menu. This method gives the agency more control over the rostering process and provides more opportunities to develop rosters that are more costeffective. Conversely, cafeteria style rostering often leads to "leftover" runs that cannot be legally combined and require costly part-time and overtime work. It can also lead to an excessive number of runs with non-consecutive days off.

Even though the agency developed rostering is likely to be more cost effective, some agencies continue to employ cafeteria style bidding. There are two potential reasons. First, it may be required by labor agreement. Secondly, some agencies believe that operator morale is higher when they get to select their own daily runs and therefore this could translate into improved attendance, fewer accidents, reduced worker compensation claims, and fewer customer complaints.

Automated scheduling software also contains various cost-effective rostering techniques, the use of which will depend upon agency policy and work rules. If an agency uses cafeteria style bidding, there is little benefit seen from using the software. However if an agency develops their weekly rosters, then the software contains many options in creating a roster that satisfies the goals and policies of the agency. Like the runcutting process, the software also allows the scheduler to run scenarios for rostering. 


\section{Scheduling Survey of Florida Transit Agencies}

\section{Methodology}

A major component of this project was a fixed route scheduling survey that was mailed to all 25 transit agencies in Florida that operate fixed route service. The survey asked a series of questions pertaining to the agency's scheduling practices and their opinions about and uses for automated scheduling software. The survey also requested that the agency attach any written operating procedures they use for their bidding process.

The survey was mailed out in early May of 2004. A total of 20 of the 25 fixed route transit agencies in Florida responded to the survey. Seven of these agencies also included their written operating procedures. A summary of the results was presented at the $3^{\text {rd }}$ Annual Florida Public Transportation Association (FPTA) / Center for Urban Transportation Research (CUTR) Professional Development Workshop in Tampa, FL on June 10, 2004. An analysis of the survey results is presented here. The actual survey sent to the transit systems is attached as Appendix A.

\section{Results}

Question \#1 asked for the method of transit scheduling that agencies utilize: manual scheduling with a spreadsheet program or by hand, or automated scheduling software. Eight agencies (MDT, BCT, JTA, LYNX, Palm Tran, PSTA, Regional Transit System - RTS in Gainesville and Polk County Transit Services) all use scheduling software, and a ninth agency (SCAT in Sarasota) has recently procured a software package and is in the implementation/testing phase. LYNX replied that they schedule using a combination of both manual and automated methods concurrently. Question \#12 further asked the agencies to specify the vendor of the scheduling software package they use. Five of the agencies are using Trapeze software products (MDT, LYNX, Palm Tran, Polk, and SCAT). A sixth agency (Hartline) also uses Trapeze (not included in the survey). Three are using GIRO HASTUS as their scheduling package (JTA, BCT, and PSTA). RTS is using a DOS-based version of a software product called Fleet-Net. Fourteen agencies are still using manual processes for some or all of their scheduling needs. 


\section{Q1. Scheduling Method and Q12. Vendor of Software Product}

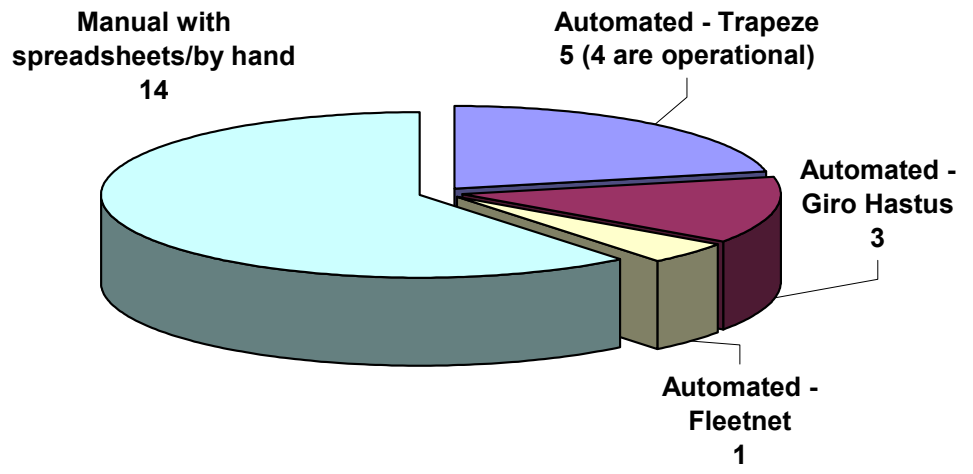

Question \#2 asked the agencies which person(s) or departments manage their transit scheduling process. The most common answer (10 out of 20,50 percent), was Operation Supervisor(s). This is even more the case for those agencies that schedule manually ( 9 out of 13, 69 percent). Specific Transit Scheduler(s) or a Scheduling Department was the answer for eight of the agencies, including (5 out of 7, 71 percent) that use automated scheduling software. Planners or Planning Departments have a role in five agencies, but only one agency responded that their Planning Department has sole responsibility for scheduling (Palm Tran). Management was cited as the agency in charge of scheduling for four agencies, all of which are small-size agencies with less than 35 buses operated in maximum service. The results of this question total more than 20 because seven agencies claimed that multiple departments were responsible for scheduling tasks.

\section{Q2. Who manages your transit scheduling?}

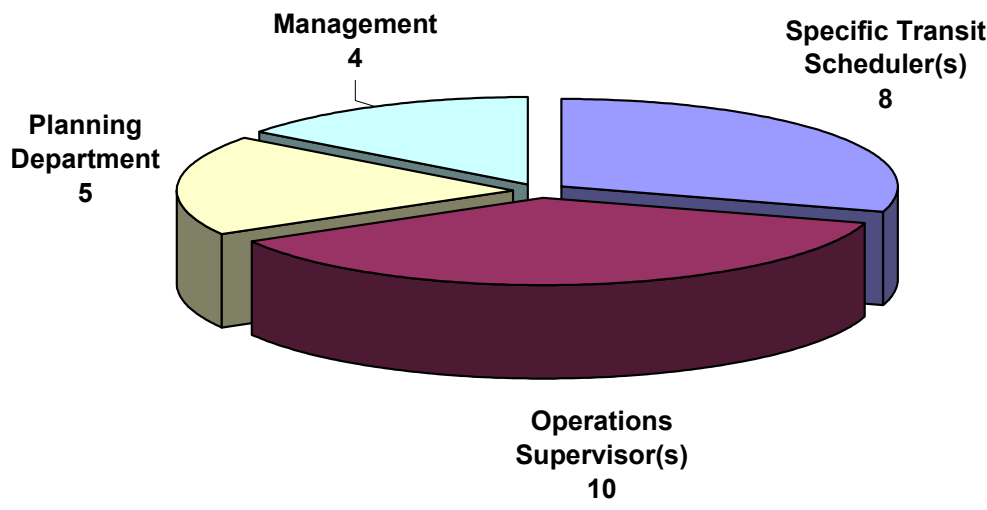


The next series of questions, \#3 through \#7, inquired about the agencies' bidding processes (pick, mark-up). Question \#3 asked which method of rostering was utilized by the agency: agency developed (weekly packages) or operator developed (cafeteria style). Agency developed rostering is the most common with nearly half ( 9 out of 20) agencies utilizing that method. Cafeteria style is used at only four transit systems. It is interesting to note that three of the four systems that utilize cafeteria style rostering (LYNX, JTA, Palm Tran) are among the systems in Florida that employ the largest number of operators (all more than 290). The fourth system, VOTRAN in Volusia County, employs 107, which classifies it as a mid-size agency. Three systems (Pasco County Public Transportation - PCPT, RTS and Key West Transit) did not specify which method they use. The remaining four systems (Bay Town Trolley, St. Lucie Council on Aging, Polk, and Indian River Council on Aging) stated that they did not have an established bidding process for assigning their operators a weekly schedule.

\section{Q3. Method of Rostering}

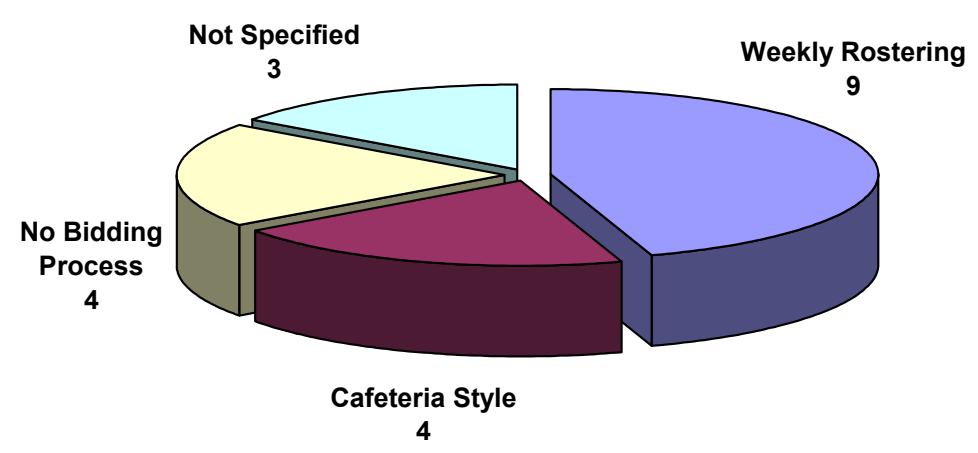


Question \#4 asked the agencies how many bidding periods they execute per year. The most frequent period of once every quarter (three months), is used by five agencies. Ten systems run their bid process three times per year. Two systems (Key West and SCAT - Sarasota) execute their bid process on a bi-annual basis. A few agencies gave a range (2-3 or 3-4 times per year) and those results are reflected in both columns, resulting in a response total greater than 20 .

\section{Q4. Bids per Year}

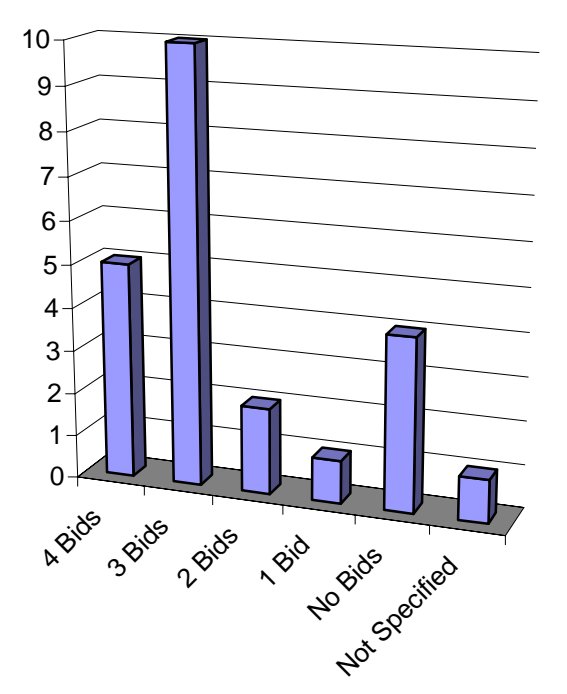


Question \#5 asked if the number of bids per year was based upon the labor agreement (union contract) or was an agency self-imposed rule. Of the sixteen agencies that replied that they have a bid process, nine of them said their number of bids was based on their labor agreement and the other seven said it was based on an agency rule. Question \#7 was related to question \#5 as it asked how much of an impact does the labor agreement have on the scheduling process overall. The options provided on the survey were: completely dictates the schedule, has a substantial impact, some adjustments are required, has only a slight impact, and no impact at all. None of the agencies answered that the labor agreement completely dictated the schedule. However, five agencies responded that it had a substantial impact (MDT, BCT, JTA, PSTA, and RTS). It is significant to note that all five of these agencies also use automated scheduling software. Only one agency said that some adjustments must be made (Key West). Four agencies (LYNX, SCAT-Sarasota, Palm Tran, and Escambia County Area Transit - ECAT) all stated that the labor agreement only has a slight impact on scheduling. The remaining six agencies that answered question \#7 said that the labor agreement had no impact on scheduling. All of these agencies are in the small to mid-size range in terms of operators and vehicles.

\section{Q5. Bids per Year based on and Q7. Impact of Labor Agreement}

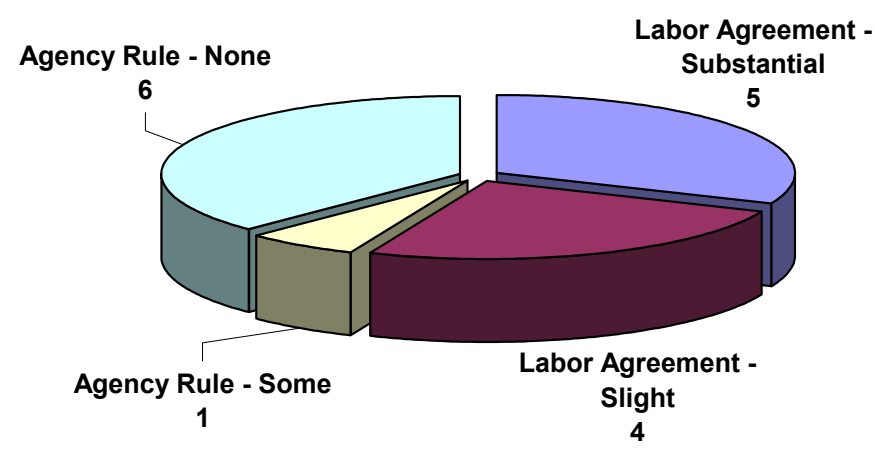


Question \#6 asked a simple yes/no question - does your agency have written operating procedures for your bidding process? Thirteen agencies (65 percent) responded yes, and only two agencies responded no. The four agencies that claimed they have no bidding process remained consistent by not answering this question.

\section{Q6. Written Operating Procedures for Bid Process?}

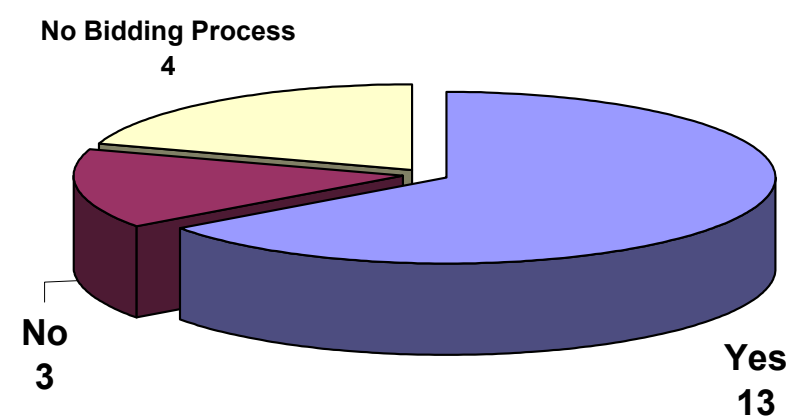

Question \#6 also requested that if an agency answered yes to the question, that they attach a copy of their written operating procedures with their submittal. Seven of the thirteen agencies complied with this request (BCT, JTA, PSTA, SCAT - Sarasota, ECAT, LeeTran and Pasco). The first five of these systems sent the applicable sections of their labor agreement pertaining to the bid process. The rules and procedures for each agency varied in length, with BCT, JTA and ECAT's quite lengthy while SCAT and PSTA had more concise rules. The remaining two systems (LeeTran and Pasco) had agency created rules and procedures for the bid process. Both of these were very short at less then two pages in length. 
Question \#8 asked the agencies to rate a list of characteristics based on the difficulty they have accounting for them in their scheduling process. They were instructed to rate each characteristic on a scale from ( 1 to 5 ) with 1 defined as no difficulty and 5 defined as a major difficulty. The characteristics listed were layover times, deadhead times, running times, transfer location times, relief locations, operator assignment to vehicles (runcutting), and operator weekly scheduling (rostering). The results are shown in the following graphic, Q8. Running times were ranked the most difficult with an average score of 2.35 , followed closely by rostering at 2.32 . Deadhead times were cited as the least difficult characteristic to manage in the scheduling process with an average score of 1.6. An average score of 3 would signify moderate difficulty, yet none of the characteristics approached this score, indicating that none of the characteristics posed much difficulty overall. However, scores varied widely for each individual agency based on the number of vehicles operated in maximum service (VOMS) and the use of automated scheduling software. Florida's three largest transit systems in terms of number of operators and buses (MDT, BCT, and LYNX) all expressed moderate difficulty in at least three categories and in the case of MDT (by far the largest system), moderate to major difficulty in all seven categories. This is the case despite all three agencies utilizing the capabilities of automated scheduling software. The next four largest Florida transit systems that completed the survey (JTA, PSTA, Palm Tran, and RTS) also use automated scheduling software and on average expressed very little difficulty. This suggests that as agencies grow, there is a threshold size where scheduling difficulties increase even with the aid of automated scheduling. On the other hand, the results also indicate that agencies that utilize automated scheduling software have fewer difficulties on average then agencies that do not. This held true for six of the seven categories, with the lone exception being runcutting. Grouping the Florida Transit Systems into four groups (very large $=>250$ VOMS, large $=100-250$ VOMS, medium $=25-100$ VOMS, and small $=$ $<25$ VOMS) reveals some additional findings. The only very large agency (MDT) had at least moderate difficultly in all seven categories; the large agencies (BCT, LYNX, JTA, PSTA, and Palm Tran) had the most difficulty with running times and transfer location times; the mid-sized agencies (RTS, Taltran in Tallahassee, VOTRAN, LeeTran in Lee County, Citrus Connection in Lakeland, SCAT - Sarasota, and ECAT) had the most difficulty with rostering; and the small-sized agencies (Pasco, Indian River, Bay Town Trolley, Key West, Sun Tran in Ocala, Polk County, and St. Lucie COA) had the most difficulty with layover times, running times, and relief locations. These varied results indicate that scheduling difficulties can be very different for an agency depending on the size of its fleet and operations. 


\section{Q8. Difficulties in Scheduling}

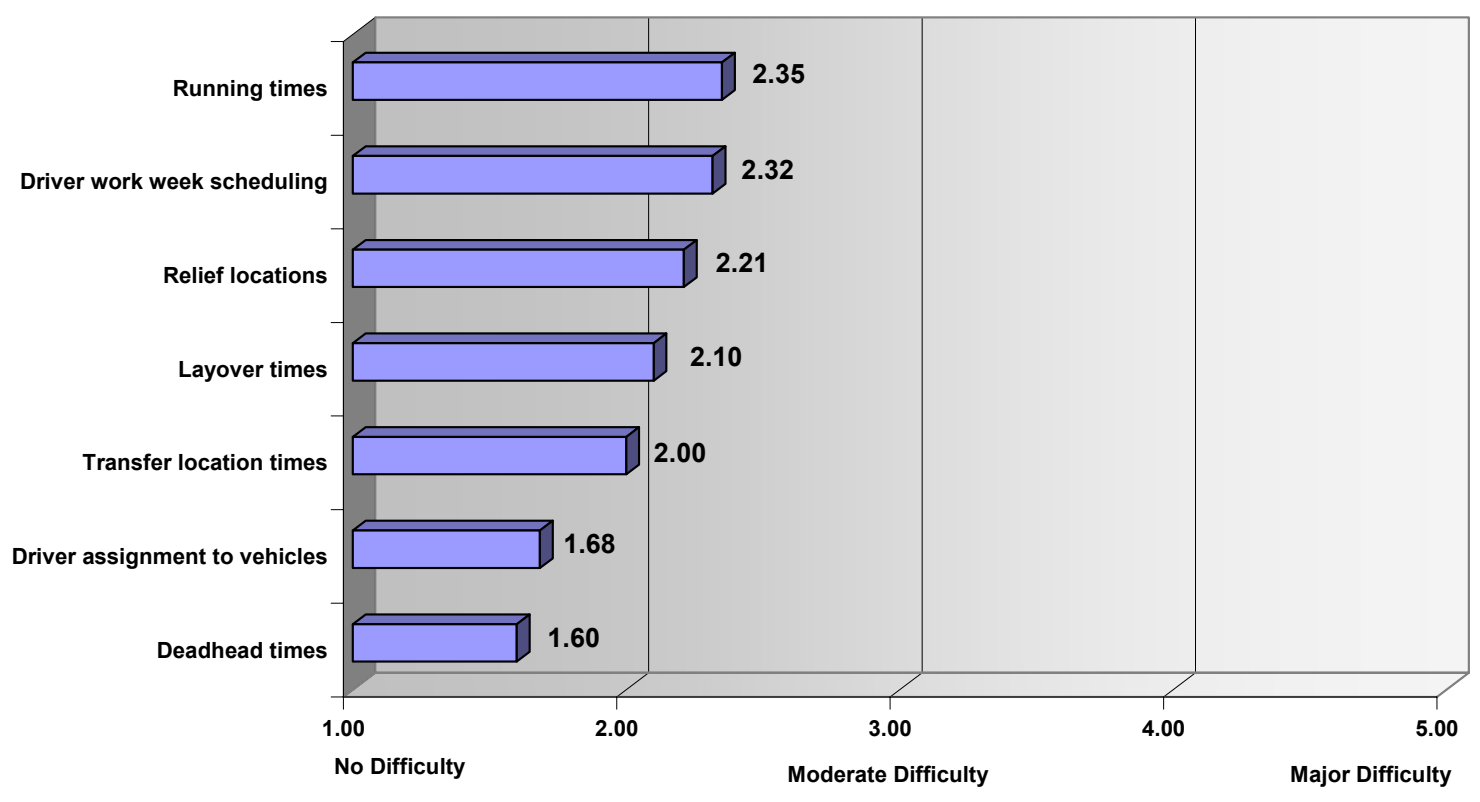


Question \#9 asked for the level of satisfaction that the agency had with their scheduling practices. The purpose of this question was to gauge the interest of the agencies in improving their scheduling processes. Only one agency (Citrus Connection) was completely satisfied with their scheduling practice. Of significant importance is the fact that half of the agencies who responded (10 out of 20) stated that they were only somewhat satisfied with their scheduling and feel there is room for improvement. A majority of these agencies (6 out of 10) are not currently using automated scheduling software. Another two agencies expressed that they are definitely looking for a better way to schedule their services (neither of these uses automated scheduling software).

\section{Q9. Level of Satisfaction with Scheduling Practices}

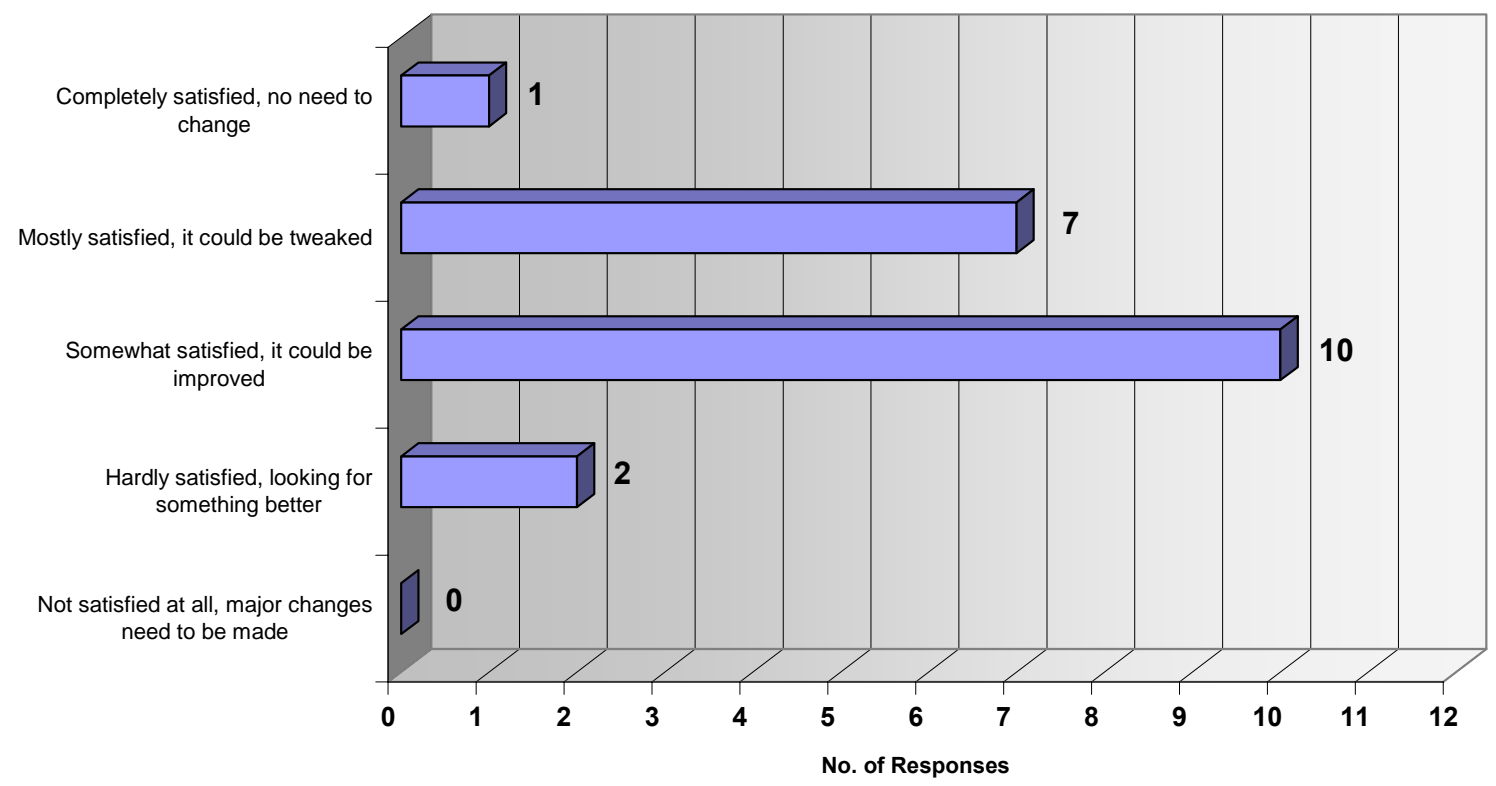


Question \#10 asked agencies for the largest challenges they face in regards to conversion from manual scheduling to using automated scheduling software. Software costs ranked as the most challenging factor at 3.36. Hardware costs and resistance to change both scored as a moderate challenge (3). Lack of staff expertise and lack of training were the least likely challenges but with scores above two, neither category can be ignored. Clearly, the costs of upgrading to automated scheduling are a barrier for small to medium transit systems. A potential solution to the cost issue would be the purchase of a software package by CUTR, which in turn would provide scheduling services to these agencies. Question \#11 on the survey addresses this concept.

\section{Q10. Challenges for Converting to Automated Scheduling Software}

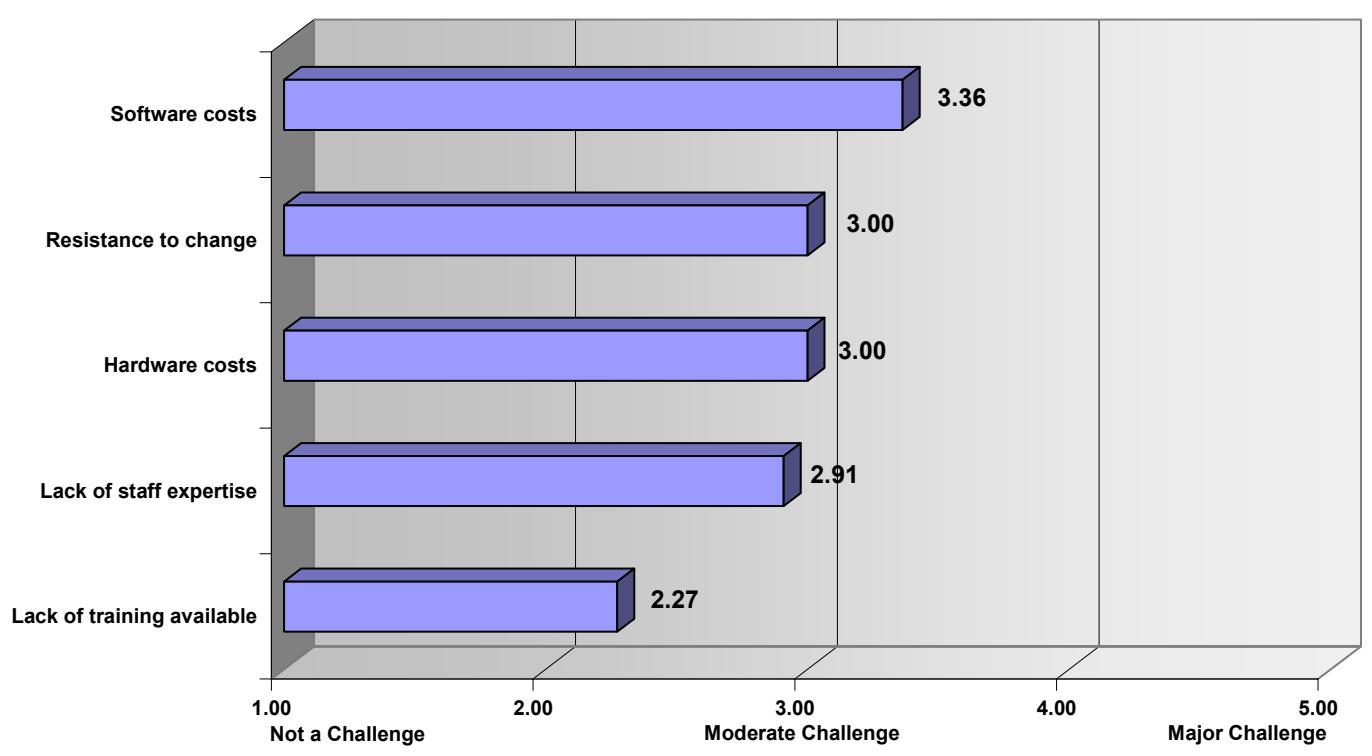


Question \#11 was a theoretical question aimed at gauging agency's interest in altering their current scheduling practices. The question asked agencies if they would be interested in using automated scheduling software if CUTR purchased a software package and was designated as a resource center that could provide scheduling functions and support for their agency. Along with question $\# 9$, these two questions together provide a good indication of the level of interest. This question had four possible yes answers (all facets of the scheduling process, blocking, runcutting, and rostering). The no answer stated that the agency was completely satisfied with their current methods. Finally, a maybe answer stated that the agency would need more information before deciding.

Not unexpectedly, the agencies that already have a scheduling software package in place are not interested in surrendering their current scheduling practices. The lone exception is LYNX, who may have interpreted the question differently from the rest. An additional choice should have been provided for those agencies who have automated scheduling software that may want training or assistance on how to maximize the use of the software. Because this question was not asked, the level of interest for assistance among systems using automated software is unknown at this time. More promisingly is the fact that all agencies that do not use an automated scheduling software package answered with either a yes or maybe answer. Among the medium-sized systems, one agency answered yes and four agencies answered maybe. Among the small-sized properties, four agencies answered yes, two agencies answered maybe, and one agency did not provide an answer. Based on these results, it is apparent that there is a general interest among small to medium sized transit agencies in the State of Florida in pursuing the benefits of fixed route automated scheduling.

Further affirmation of interest is evident in a write-in response from the Indian River Council on Aging, which is classified as a small system based on the size of its fixed route fleet; however the respondent refers to it as a medium sized system.

"The problem for medium sized systems is the software is highly expensive and updated hardware is usually needed thus increasing the cost even more. If CUTR could purchase and provide automated software at a reasonable cost, many more rides could be provided with the cost saving features of the software. This is a great idea and so needed by us mid-sized systems." 
The next series of questions, \#13 through \#17, asked for answers from only those agencies that use automated software for their scheduling needs. Question \#13 asked for the names of the specific modules that the agencies utilize. Of those agencies using Trapeze, all of them are utilizing FX which is the standard package for fixed route services. Palm Tran is also using Trapeze's PLAN (advanced tools for ridership analysis, forecasts and reports) and OPS (tools to manage bidding, driver dispatch, timekeeping and workforce management). Miami Dade Transit is considering an upgrade to Trapeze OPS in the near future. Currently MDT uses an older software product made by Teleride Sage called the Transit Operations System (TOS) for their dispatching, bidding, and timekeeping processes. All of these agencies are using the Windows version of FX, except for LYNX who is still utilizing the DOS version, although they have plans to upgrade to the Windows version soon. Of the agencies using GIRO HASTUS, JTA uses HASTUS-Vehicle for trip building and HASTUS-Crew and CrewOpt for runcutting. BCT also uses these modules as well as HASTUS-Roster for rostering and HASTINFO for customer service. PSTA uses all of the above modules except for HASTINFO. They also are the only agency to use HASTUS-DDAM, which is a module for dispatchers to track transit operator assignments on a daily basis. 
Question \#14 inquired about the various uses of automated scheduling software. Seven out of eight agencies utilize the software for their blocking and runcutting needs. The only exception is Polk, which only uses their software for mapping and customer service purposes. Five of the agencies use their software for rostering (MDT, BCT, RTS, PSTA and Palm Tran). Polk, LYNX, and JTA continue to use manual rostering. Five agencies use their software for customer service and four agencies use it for mapping purposes. Three agencies integrate their scheduling software with their financial system to pay their operators. PSTA has plans to implement this integration in FY 2005. Three other uses were write-in answers listed below. See Table 1 for complete results.

\section{Table 1-Q14. Scheduling Software Uses}

\begin{tabular}{|l|c|c|c|c|c|c|c|c|c|}
\hline \multicolumn{1}{|c|}{ Uses } & RTS & JTA & PSTA & $\begin{array}{c}\text { Palm } \\
\text { Tran }\end{array}$ & LYNX & Polk & BCT & MDT & Totals \\
\hline Blocking & $\sqrt{ }$ & $\sqrt{ }$ & $\sqrt{ }$ & $\sqrt{ }$ & $\sqrt{ }$ & & $\sqrt{ }$ & $\sqrt{ }$ & $\mathbf{7}$ \\
\hline Runcutting & $\sqrt{ }$ & $\sqrt{ }$ & $\sqrt{ }$ & $\sqrt{ }$ & $\sqrt{ }$ & & $\sqrt{ }$ & $\sqrt{ }$ & $\mathbf{7}$ \\
\hline Rostering/Bidding & $\sqrt{ }$ & & $\sqrt{ }$ & $\sqrt{ }$ & & & $\sqrt{ }$ & $\sqrt{ }$ & $\mathbf{5}$ \\
\hline Customer Service & & & $\sqrt{ }$ & $\sqrt{ }$ & & $\sqrt{ }$ & $\sqrt{ }$ & & $\mathbf{4}$ \\
\hline Mapping & & $\sqrt{ }$ & $\mathbf{x}$ & $\sqrt{ }$ & & & & $\sqrt{ }$ & $\mathbf{3}$ \\
\hline Integrated with financial system - pay operators & & & & & & & & & $\mathbf{1}$ \\
\hline Other - Maintenance management & & $\sqrt{ }$ & & & & & & & $\mathbf{1}$ \\
\hline Other - Transfer run changes & & & & & & & & $\sqrt{ }$ & $\mathbf{1}$ \\
\hline $\begin{array}{l}\text { Other - Integrated with other software - } \\
\text { MIDAS, Smart Trac, Corel }\end{array}$ & & & & & & & $\sqrt{ }$ & & \\
\hline
\end{tabular}

$\mathrm{x}$ - to be implemented in 2005 
Question \#15 asked what benefits the agencies experienced from using automated scheduling software. A list of potential benefits was provided on the survey, with the option to write in other benefits not listed. All eight agencies stated that using automated scheduling software sped up the scheduling process (saved time) and was an easier process than manual scheduling. Flexibility was a benefit for six agencies and cost savings were achieved at six agencies. The reduction of operators was a benefit for four agencies, and the reduction of vehicles was a benefit for three agencies. There were three write-in answers listed below. Table 2 shows the complete results.

\section{Table 2 - Q15. Scheduling Software Benefits}

\begin{tabular}{|c|c|c|c|c|c|c|c|c|c|}
\hline Benefits & RTS & JTA & PSTA & $\begin{array}{l}\text { Palm } \\
\text { Tran }\end{array}$ & LYNX & Polk & BCT & MDT & Totals \\
\hline Process is faster than manual (save time) & $\sqrt{ }$ & $\sqrt{ }$ & $\sqrt{ }$ & $\sqrt{ }$ & $\sqrt{ }$ & $\sqrt{ }$ & $\sqrt{ }$ & $\sqrt{ }$ & 8 \\
\hline Process is easier than manual & $\sqrt{ }$ & $\sqrt{ }$ & $\sqrt{ }$ & $\sqrt{ }$ & $\sqrt{ }$ & $\sqrt{ }$ & $\sqrt{ }$ & $\sqrt{ }$ & 8 \\
\hline Process is more flexible & & $\sqrt{ }$ & $\sqrt{ }$ & & $\sqrt{ }$ & $\sqrt{ }$ & $\sqrt{ }$ & $\sqrt{ }$ & 6 \\
\hline Cost savings achieved & $\sqrt{ }$ & $\sqrt{ }$ & & $\sqrt{ }$ & $\sqrt{ }$ & & $\sqrt{ }$ & $\sqrt{ }$ & 6 \\
\hline Reduced drivers needed & $\sqrt{ }$ & $\sqrt{ }$ & $\sqrt{ }$ & & & & $\sqrt{ }$ & & 4 \\
\hline Reduced vehicles needed & $\sqrt{ }$ & & $\sqrt{ }$ & & & & $\sqrt{ }$ & & 3 \\
\hline $\begin{array}{l}\text { Other - build schedules to make } \\
\text { corrections on multiple routes }\end{array}$ & & $\sqrt{ }$ & & & & & & & 1 \\
\hline Other - overtime control/reduction & & & & & $\sqrt{ }$ & & & & 1 \\
\hline Other - improved interlining opportunities & & & & & $\sqrt{ }$ & & & & 1 \\
\hline
\end{tabular}

Question \#16 asked the agencies if they utilize their scheduling software to run scenarios. Scenarios can be defined as different possible scheduling solutions. By running scenarios, an agency can find a better solution for their schedules. Five agencies said that they run scenarios (BCT, JTA, LYNX, PSTA and Palm Tran) and three do not (MDT, RTS and Polk).

Question \#17 asked for those agencies that use automated scheduling software to list any specific observations or measured benefits that they have achieved. RTS, which uses FleetNet, claims that the software provides a better way to keep driver's time, eliminates re-bids due to manual errors, and provides more accurate information. LYNX, a Trapeze FX user, states that the software enabled them to analyze daily operator costs for run cut scenarios, increase their ability to cut "straight" runs with no additional operating costs, and cut back on the number of split runs (from 50 percent to under 20 percent). MDT, which also uses Trapeze software, sites the benefits of 
standardization of running times by route, more valid data, and greater availability of reports. Polk claims better customer service and better accuracy of bus stop locations from using Trapeze. BCT, which uses GIRO HASTUS, achieved a reduction of nine operator positions after two run cuts using the software. BCT also cited a reduction in time scheduling relief vehicles. What used to take them two weeks can now be accomplished in 2-3 days. HASTUS integrated reports help to eliminate erroneous information distributed among the BCT staff. JTA, which also uses HASTUS, is the subject of an application overview in this report and their observations and benefits are displayed in greater detail in that section. Hartline is the transit system chosen for a case study of an agency using Trapeze.

Table 3 on the following page is a summary table that provides a comparison of agency's answers for many of the questions on the survey. The agencies are color coded into four groups (very large, large, medium, and small) based on the number of vehicles operated in maximum service (VOMS). Taltran and Bay Town Trolley both specified that they use part time operators and this is reflected on the table with the abbreviation PT. The abbreviation FT signifies their full-time operators. 
Table 3 - Scheduling Survey Summary Table

\begin{tabular}{|c|c|c|c|c|c|c|c|c|c|c|c|c|c|c|c|c|c|}
\hline \multirow[b]{2}{*}{ Transit Agency Name } & \multirow{2}{*}{ Operators } & \multirow{2}{*}{$\frac{\frac{\text { Veh. Oper. in }}{\text { Max. Service }}}{\text { (VOMS) }}$} & \multirow{2}{*}{$\frac{\frac{\text { Automated }}{\text { Scheduling }}}{\text { Software }}$} & \multirow[b]{2}{*}{ Department } & \multirow{2}{*}{$\frac{\begin{array}{r}\text { Labor } \\
\text { Agreement }\end{array}}{\text { Impact }}$} & \multicolumn{6}{|c|}{$\underline{\text { Scheduling Difficulties }}$} & \multicolumn{6}{|c|}{ Sched Challenges } \\
\hline & & & & & & & & & & & Ro & Satisfaction & LE & & & & Resource Center? \\
\hline$\overline{\text { MDT }}$ & 1646 & 688 & $\overline{\text { Trapeze }}$ & $\mathrm{TS}$ & Substantial & $\overline{3}$ & 3 & 4 & 3 & $\sqrt{3} \overline{5}$ & $\overline{3}$ & Mostly & $\overline{5}$ & 5 & $\sqrt{3} \overline{3}$ & $\overline { 3 } \longdiv { 5 }$ & $\mathrm{No}$ \\
\hline Broward County Transit & 580 & 210 & Hastus & TS & Substantial & 3 & 1 & 3 & 3 & 2 & 3 & Mostly & 3 & 2 & 1 & 2 & No \\
\hline LYNX & 544 & 197 & Trapeze & TS, P & Slight & 4 & 1 & 4 & 3 & 1 & 1 & Mostly & 4 & 2 & 2 & 4 & Maybe \\
\hline Jacksonville Transit Authority & 350 & 144 & Hastus & TS & Substantial & 1 & 1 & 1 & 1 & 1 & 1 & Somewhat & 1 & 1 & 5 & 5 & No \\
\hline PSTA & 342 & 142 & Hastus & TS & Substantial & 1 & 1 & 3 & 1 & 1 & 1 & Mostly & & & & & No \\
\hline PalmTran & 292 & 105 & Trapeze & $\mathrm{P}$ & Slight & 1 & 1 & 1 & 3 & 3 & 1 & Mostly & & & & & No \\
\hline Hartline & 275 & 171 & Trapeze & TS & & & & & & & & & & & & & \\
\hline Regional Transit System (RTS) & 152 & 88 & Fleet-Net & OS, P & Substantial & 1 & 1 & 1 & 2 & 4 & 1 & Somewhat & & & & $\mathrm{x}$ & No \\
\hline Taltran & 78 FT, 30 PT & 48 & None & OS, P & None & 2 & 2 & 2 & 1 & 1 & 4 & Somewhat & 2 & 2 & 1 & 1 & Maybe \\
\hline VOTRAN & 107 & 45 & None & TS & None & 3 & 1 & 1 & 1 & 1 & 1 & Somewhat & & & & & Maybe \\
\hline LeeTran & 115 & 44 & None & os & None & 1 & 1 & 2 & 3 & 1 & 4 & Hardly & 3 & 1 & 3 & 3 & Yes - RC, Rostering \\
\hline Citrus Connection (LAMTD) / WHAT & 80 & 33 & None & M & None & 1 & 2 & 2 & 1 & 1 & 5 & Completely & & & & & Maybe \\
\hline Sarasota County Area Transit & 101 & 26 & Trapeze & os & Slight & 1 & 1 & 1 & 1 & 1 & 4 & Somewhat & 5 & 5 & 1 & 1 & No \\
\hline Escambia County Area Transit (ECAT) & 53 & 25 & None & os & Slight & 1 & 1 & 2 & 3 & 2 & 2 & Mostly & 4 & 3 & 5 & 1 & Maybe \\
\hline Pasco & 30 & 14 & None & M & None & 2 & 2 & 4 & 3 & 3 & 3 & Somewhat & 1 & 1 & 3 & 3 & Yes - RC, Rostering \\
\hline Indian River & 12 & 9 & None & TS & N/A & 4 & 3 & 2 & 2 & 1 & 2 & Hardly & $\mathrm{x}$ & & & $\mathrm{x}$ & Yes \\
\hline Bay Transportation - Bay Town Trolley & $14 \mathrm{FT}, 4 \mathrm{PT}$ & 6 & None & OS, P & N/A & 1 & 1 & 4 & 3 & 1 & 2 & Mostly & & & & & N/A \\
\hline Key West Department of Transportation & 15 & 6 & None & OS, TS & Some & 5 & 4 & 3 & & 1 & & Somewhat & $\mathrm{x}$ & $\mathrm{x}$ & $\mathrm{x}$ & $\mathrm{x}$ & Probably Yes \\
\hline SunTran & 15 & 6 & None & os & None & 1 & 1 & 1 & 1 & 1 & 1 & Somewhat & 2 & 2 & 5 & 5 & Maybe \\
\hline Polk County Transit Services & 11 & 4 & Trapeze & M & $\mathrm{N} / \mathrm{A}$ & 1 & 1 & 2 & 1 & 3 & 3 & Somewhat & & & & & Maybe \\
\hline St. Lucie COA & 6 & 3 & None & M & N/A & 5 & 3 & 4 & & 1 & 2 & Somewhat & 2 & 1 & 4 & 5 & Yes - Runcutting \\
\hline Space Coast Area Transit & ? & 17 & None & & & & & & & & & & & & & & \\
\hline MCAT & ? & 13 & None & & & & & & & & & & & & & & \\
\hline Okaloosa & ? & 3 & None & & & & & & & & & & & & & & \\
\hline Collier & $?$ & $?$ & None & & & & & & & & & & & & & & \\
\hline
\end{tabular}

Very Large $=>250$ VOMS

Large $=100$ to 250 VOMS

Medium $=25$ to 100 VOMS

Small $=<25$ VOMS

Agencies in italics have no survey results.

Blanks or N/A indicate that no answer was given for that particular question.

An $\mathrm{x}$ signifies that the agency did not provide a number ranking, but selected this answer with an $\mathrm{x}$.

An $\mathrm{x}$ answer had no impact on the calculated average scores. 
To better understand how agencies are utilizing automated scheduling software, three agencies were selected for application overviews that provide a summary of their use. A history of scheduling at these three agencies is described, what decisions prompted them to convert to automated scheduling, what they use the software for, and what benefits they have realized. Cost information was included when available. The three systems chosen were JTA, which uses GIRO HASTUS; Hartline, which uses Trapeze, and RTS, which uses Fleet-Net.

\section{Application Overview: GIRO HASTUS - JTA}

The Jacksonville Transportation Authority in Duval County, FL is an example of a fairly large transit system that has upgraded their transit scheduling software recently. JTA used manual scheduling processes until 1988, when they purchased a DOS-based software package from Teleride Sage called G/Sched. This program was capable of automating the trip building and blocking tasks of scheduling, but did not have runcutting capability. JTA continued with manual runcuts until 1994 when they purchased a $\$ 65,000$ add-on called GSched Optimizer that could automate the runcutting process. In early 2001, JTA decided that they needed an up-to-date and robust scheduling package with more functionality for their growing fixed route system. JTA issued a Request for Proposal (RFP) and received three bids: GIRO HASTUS, Trapeze, and Teleride Sage (who has since been bought out by VERSYSS). GIRO HASTUS was selected with the low bid and the software was accepted and put into operation in May 2003 after a nine month installation period. The cost of the software is based upon the number of vehicles in peak service. The total cost included costs for project management, specifications, training, customization, implementation support, configuration and installation, algorithm calibration, expenses, and an extended warranty. The total cost for JTA (based on 160 vehicles in maximum service) for the HASTUS scheduling and runcutting module was $\$ 240,534$. The mapping module (GEO) and the stop poster module (HASTOP) were offered to JTA in the RFP, but were declined due to cost restrictions. The costs for these modules were $\$ 53,775$ and $\$ 46,986$ respectively. JTA is considering purchasing additional modules of HASTUS in the future including: HASTINFO for customer service applications, HASTUS-DDAM for day-to-day management and GIRO/ACCES for paratransit scheduling. JTA decided not to purchase the rostering module from HASTUS and continues to use a manual bidding process. They do not have plans to purchase the HASTUS module for this function due to their current cafeteria style bidding approach. 
Maintenance costs for HASTUS are priced per module and are paid the first year after the warranty period. The prices are subject to annual cost of living increases, currently set at $2.5 \%$ per year. The 2001-2002 maintenance cost for the scheduling and runcutting modules was quoted at $\$ 16,112$ in the JTA proposal. JTA paid extra to extend the standard three-month warranty to twelve months and therefore their maintenance payments did not begin until May 2004. GIRO also charges JTA for changes made to the software after installation at a rate of $\$ 125$ per hour.

Since installation, JTA has observed several advantages of HASTUS over its previous software. The number of time points that can be saved in HASTUS is unlimited, while the maximum allowed in Teleride Sage was 20. This is extremely important for an agency the size of JTA. Another major advantage is the ability to make changes to HASTUS rules directly from the program menus. With Teleride Sage, the program change had to be requested via phone and Teleride would have to make the change and send it to JTA on disk. JTA also saves valuable staff time during the runcutting process. The program can perform JTA's runcutting process for 2000 platform hours in as few as ten minutes. Finally, JTA has been able to eliminate two operator positions to achieve an annual cost savings of approximately $\$ 65,000$.

From a planning prospective, the biggest benefit is the ease of conducting "what if" scenarios. It is easy to create a "plan" set of data, adjust a set of schedules, and calculate the impact on the fleet and operator pay time. The previous software, G/Sched, was not a networked application, meaning JTA would have to run a complete new schedule set, and could not run it "on-line" while running other applications, or if in the middle of an active bid. Additional planning applications are available with other modules yet to be purchased. HASTUS-GEO, the mapping module, is capable of planning routes and the JTA Planning Department sees potential in the possible integration of APC data. 


\section{Application Overview: Trapeze - Hartline}

Hartline in Tampa, FL is another example of a fairly large transit system that has upgraded their scheduling software. Hartline, like JTA, was a user of Teleride Sage's G/Sched. In the year 2000, they decided they wanted a system that would have a positive effect on the entire organization and was compatible with the Windows Environment. Trapeze was purchased after it was selected with the low bid and met the capabilities that Hartline was looking for. The other two vendors to bid were GIRO HASTUS and Multisystems. Multisystems has since been bought out by Trapeze. Hartline did not go live with the software until 2002, due to a lengthy installation process that was marred by glitches. The module first installed was the basic scheduling and runcutting software entitled FX. Other modules purchased were OPS (dispatching), INFO (customer service), PLAN (planning tools), PASS (paratransit scheduling), and FLEX (route deviation scheduling). Table 4 shows the initial costs (including installation and project management costs) and the maintenance costs associated with each module.

Table 4 - Trapeze Costs at Hartline

\begin{tabular}{|l|l|l|l|l|}
\hline Module & Initial Cost & $\begin{array}{l}\text { Maintenance Costs } \\
\text { per Year (Current) }\end{array}$ & $\begin{array}{l}\text { Vehicle/Trip } \\
\text { Limits }\end{array}$ & Workstations \\
\hline FX & $\$ 118,000$ & $\begin{array}{l}\$ 15,100 \text { original } \\
\$ 22,100 \text { thru Sep 04 } \\
\$ 2,100 \text { Sep 04-05 }\end{array}$ & 200 Vehicles & 3 \\
\hline OPS & $\$ 189,500$ & $\$ 22,500$ & 200 Vehicles & 5 \\
\hline INFO & $\$ 128,000$ & $\$ 24,850$ & 199 Vehicles & 8 \\
\hline PLAN & $\$ 43,000$ & $\$ 7,400$ & 200 Vehicles & 2 \\
\hline PASS & $\$ 64,000$ & $\$ 20,650$ & 1599 Trips/day & 10 \\
\hline FLEX & $\$ 49,000$ & $\$ 12,600$ & 400 Trips/day & 2 \\
\hline $\begin{array}{l}\text { Malteze } \\
\text { interface with } \\
\text { payroll) }\end{array}$ & $\$ 10,000$ & $\$ 5,500$ & N/A & N/A \\
\hline Escrow & $\$ 2,000$ & $\$ 1,600$ & N/A & N/A \\
\hline Total & $\$ \mathbf{6 0 3 , 5 0 0}$ & $\$ 114,200$ & N/A \\
\hline
\end{tabular}


The costs shown are based on the number of vehicles for fixed route services and on number of trips for paratransit and route deviation services. Also factoring into the costs is the number of workstations the software is installed on. Maintenance costs increase each year at a much higher rate than cost of living based on the figures given for the FX module. Any customization reports cost a flat fee of $\$ 10,000$ such as the interface with payroll that Hartline requested. Other costs associated with Trapeze include a mapping upgrade charge of $\$ 1500$ per county. An agency also has the option of purchasing a mapmaker module costing between $\$ 15,000$ and $\$ 30,000$ that enables a staff person the capability of updating the map without incurring a charge each time an update is made. Due to the mapping expertise needed for this task, Hartline decided not to purchase this capability and pays the $\$ 1500$ for each update.

Comparing the costs of Trapeze with GIRO HASTUS, it is apparent why Trapeze is the most used fixed route scheduling software in the Florida market. JTA paid $\$ 228,450$ for HASTUS scheduling and runcutting software excluding the extended warranty for a 160 vehicle fleet. Hartline paid only $\$ 118,000$ for the comparative Trapeze FX module for a 200 vehicle fleet. However, HASTUS does allow for unlimited licenses and unlimited installations. The price that Hartline paid limited the use of the software to three workstations. This will not likely be an issue for small to medium transit systems as they will not likely need to install the software on multiple machines. Likewise, if CUTR was to purchase a scheduling software package, the unlimited licensing would likely not be a factor in the decision on whether or not to purchase HASTUS.

Hartline's satisfaction with Trapeze depends on which department you ask. The Scheduling Department, which consists of four staff members (1 Transit Operations Analyst II, 2 Transit Operations Analyst I, and 1 Transportation Assistant), has been mostly pleased with the software and its capabilities. They acknowledged the excessive length of the installation period and attributed it to the lack of knowledge base of the Trapeze specialists. However, they claimed that as the specialists have become more experienced with the software, the customer service has dramatically improved. On a scale of 1-10, the scheduling department gave Trapeze a 7.5 stating that it provides what they need. Some benefits that were mentioned by the staff were that the software was faster than G/Sched and was also more accurate. This enables the staff to complete the bidding process in a more timely fashion. The current method of bidding is weekly rostering, which replaced the cafeteria style bidding in January 2003. Hartline is creating manual rosters, due to the automatic rostering tool in Trapeze not working correctly. Trapeze is currently working on a fix for this problem. Hartline does 
not use the automatic runcutting tool in Trapeze, instead preferring to cut the trips manually using the software. The scheduling staff claims that they do not prefer the results of the automatic runcutting tool.

Another perspective on Trapeze was provided by a member of the Hartline Information Systems (IS) Department. Her opinion was that Trapeze is still not responsive enough to requests. She felt that GIRO HASTUS is a superior product because it's better integrated and its native environment is Windows. In contrast, Trapeze software began in DOS and the programs had to be converted to a Windows Environment. Furthermore, Trapeze has purchased other software companies and attempted to integrate their software capabilities with their current software resulting in compatibility and integration issues that are still not completely resolved. 


\section{Application Overview: Fleet-Net - RTS}

The Regional Transit System (RTS) in Gainesville, a mid-sized system, is the only agency in Florida that currently uses automated scheduling software other than Trapeze or GIRO HASTUS. Prior to 1998, RTS manually performed their scheduling processes. Manual scheduling presented various problems for RTS staff such as overlapping work assignments, grievances due to payroll and scheduling problems, incomplete NTD data collection, and frequent re-bids due to mistakes on manual bids. A renewed focus towards the University of Florida in the mid 1990's led to an increase in operators and vehicles required to provide service. RTS decided that automated scheduling software could improve its scheduling efficiencies and eliminate their problems. RTS issued an RFP in early 1998 and selected Fleet-Net as their vendor in July 1998. The most important reason for selecting Fleet-Net was that the other bids received were cost prohibitive. Fleet-Net cost approximately $\$ 100,000$ at installation with maintenance costs of roughly $\$ 15,000$ per year. RTS has been satisfied with the performance of the software as it has addressed their needs and fixed their problems. Additional benefits are an accurate employee database with Human Resource functions, easy access to historic data, better NTD data collection, and operation cost savings. On the negative side, Fleet-Net is still a DOS-based program with limitations in reporting and fleet management. Other upgrades and uses that RTS is interested in that Fleet-Net does not currently offer are webbased trip planning and passenger information, GIS mapping capabilities, integration with AVL and $\mathrm{APC}$, and recognition of FDOT regulations and union labor agreement parameters. Fleet-Net is currently in the process of upgrading their software for the Windows Environment. Depending on what new features the Windows version offers, RTS may decide to look elsewhere for its scheduling needs.

Aside from these three software packages, there are a few other companies that specialize in the transit scheduling field but as of yet have not penetrated the Florida transit market. Teleride's old G/Sched software was bought out by VERSYSS who have an updated version titled Sched21. A Canadian-based company, Schedule Masters Inc., also offers comparable scheduling software to that of Trapeze and GIRO HASTUS at a very competitive rate. 


\section{Conclusions}

The use of automated scheduling software at the larger transit systems in Florida has yielded various improvements to their scheduling process. Scheduling software is an iterative tool that provides the scheduler with greater flexibility, functionality, and control over scheduling their services. It also works to reduces mistakes, improve vehicle and operator efficiencies, reduce staff time on tedious activities, and provide better reporting capabilities.

As reflected in the fixed route scheduling survey, there is an interest among some small to medium sized transit systems in realizing the benefits of automated scheduling software. Of the eleven (11) fixed route transit systems that currently do not use automated scheduling software, five (5) agencies were interested in some form of scheduling assistance, and the other six (6) did not respond no to assistance, but rather answered that they would need more information before making a decision.

Scheduling software costs and computer hardware costs in general were viewed as the most prohibitive aspects of attaining scheduling software. The costliness of these software packages were confirmed when completing the application overviews. Small to medium sized properties in particular typically do not have the resources to justify the purchase of automated scheduling software. Furthermore, the learning curve and extensive set-up time required is a potential obstacle for small to mid-sized systems.

The application overviews examined the three software packages currently in use at transit systems in Florida. Pros and cons exist for each software package, only some of which were examined in this report. While Trapeze and GIRO HASTUS are the current leaders in transit scheduling in the state, no specific recommendation is made as to the best scheduling solution for Florida transit systems.

Based on the findings of this report, a mechanism could be explored that would enable selected small to medium sized transit systems in Florida the opportunity to provide more efficient services through automated scheduling. CUTR, as a transportation research institute that provides specific technical support to Florida transit systems, is a valuable resource that can be utilized for scheduling support. Another potentially valuable resource is the wealth of knowledge and experience that exists at Florida transit systems that currently use automated scheduling software. This knowledge base can be exploited by the creation of a peer-to-peer network or a consulting service. Lastly, systems using automated scheduling software could also benefit from additional training opportunities, especially those systems that are newer to the automated scheduling environment. 


\section{References}

TCRP Report 30, "Transit Scheduling: Basic and Advanced Manuals"

Schedule Masters, Inc. White Papers

Sitarski, Edward, "Public Transit Scheduling - Difficulties and Opportunities"

Sitarski, Edward, "Blocking and Runcutting"

Sitarski, Edward, "Rostering” 


\section{Appendix A Survey Form}




\section{Fixed Route Transit Scheduling Survey}

Please complete the following survey in its entirety. If a question is followed by ( $\sqrt{ }$ all that apply), then select as many answers as apply to your agency. This survey is for fixed route transit only; please do not include paratransit data in any of your answers. Thank you for your assistance.

Transit agency name:

Transit agency address:

Your name:

Your title:

Your phone:

Your email:

Number of staff associated with scheduling duties:

Number of fixed route transit operators in your system:

Number of fixed route buses operating in maximum service:

Name and title of person(s) responsible for scheduling and runcutting for your system:

What is your background in scheduling? (List any experience you have)

1. What method do you utilize to schedule vehicles on your fixed route service?
A. Manually with pen and paper
B. Manually with spreadsheet program
C. Scheduling software

2. How is your transit scheduling handled organizationally?
A. Specific Transit Scheduler Position(s)
B. Operation Supervisor(s)
C. Planning Department
D. Management
E. Other (please specify) 
3. What type of bidding process do you utilize?

A. Weekly rostering - operators bid on weekly work assignments

B. Cafeteria style - operators bid on daily work assignments

4. How many times per year do you execute the bid process (a.k.a. pics, lineups, etc.)?

5. The number of bids per year is based upon:
A Union contract
B. Self-imposed rule
C. Other (please specify)

6. Do you have any written operating procedures for the bid process?
A. Yes
B. No

If you answered yes, please attach these procedures with the submittal of your survey.

7. How much of an impact do union rules have on your scheduling process?
A. Completely dictates the schedule
B. Has a substantial impact
C. Some adjustments required
D. Has only a slight impact
E. No impact at all

8. Which of the following items do you have the most difficulty accounting for in your scheduling process? Rate all of the following on a scale of $(1-5,1=$ no difficulty to $5=$ major difficulty).
A. Layover times
B. Deadhead times
C. Running times
D. Transfer location times
E. Relief locations
F. Driver assignment to vehicles
G. Driver work week scheduling

9. How satisfied are you with your current scheduling practices?
A. Completely satisfied, no need to change
B. Mostly satisfied, it could be tweaked
C. Somewhat satisfied, it could be improved
D. Hardly satisfied, looking for something better
E. Not satisfied at all, major changes need to be made 
Automatic scheduling software is available to automate the tasks associated with vehicle and driver scheduling. This software typically provides more optimal solutions and can improve efficiencies and reduce costs.

10. For your agency, what are the largest challenges for converting to an automated scheduling software system? Rate all of the following on a scale of $(1-5,1=$ not a problem to $5=$ major challenge).
A. Lack of staff expertise
B. Lack of training available
C. Hardware costs
D. Software costs
E. Resistance to change
F. Other (specify)
G. Other (specify)

11. Would your agency be interested in automated scheduling software if CUTR purchased a software package and was designated as a resource center that could provide scheduling functions and support for your agency? ( $\sqrt{ }$ all that apply)

_ A. Yes, we would like CUTR to manage all facets of our scheduling process

B. Yes, we need assistance in blocking - optimally assign vehicles to trips (a.k.a. vehicle assignment)

C. Yes, we need assistance in runcutting - optimally assign drivers to vehicles

D. Yes, we need assistance in rostering/bidding - create work weeks for drivers

E. No, we are completely satisfied with our current methods

F. Maybe, we need more information before deciding

The following questions are for only those systems that are currently using automated scheduling for fixed route service:

12. Who is the vendor of the scheduling software products you use?
A. Trapeze
B. Giro Hastus
C. Versyss
D. Schedule Masters
E. Other (specify)

13. List the names of the modules that you utilize (examples: Hastus-Vehicle, Trapeze FX): 
14. Select the following uses that you utilize the software for: $(\sqrt{ }$ all that apply)
A. Blocking
B. Runcutting
C. Rostering/Bidding
D. Integrated with financial system - pay operators
E. Mapping
F. Customer Service
G. Other (specify)

15. What benefits have you experienced from using the software? ( $\sqrt{ }$ all that apply)
A. Reduced vehicles needed
B. Reduced drivers needed
C. Process is faster than manual (save time)
D. Process is easier than manual
E. Process is more flexible
F. Cost savings achieved
F. Other (specify)
G. Other (specify)

16. Do you utilize the software to run scheduling scenarios (evaluating multiple scheduling possibilities before selecting an optimal solution)?
A. Yes
B. No, don't have the time to run scheduling scenarios
C. No, don't need to run scheduling scenarios

17. List any specific observations or measured benefits that you have achieved from using automatic scheduling software: 